\title{
Tormenta de citoquinas y tormenta de información asociadas a COVID-19: consideraciones sobre el síndrome inflamatorio multisistémico en niños
}

\author{
COVID-19-related cytokine and information storm: considerations regarding \\ multisystem inflammatory syndrome in children
}

El artículo sobre síndrome inflamatorio multisistémico en niños relacionado con coronavirus disease 2019 (COVID-19) de Taffarel y col., que se publica en este número, en el que se presentan dos casos clínicos, describe la relación entre la infección por COVID y la criticidad en el paciente pediátrico. Si bien, en la población pediátrica, la infección por COVID es de características clínicas leves-moderadas, el síndrome inflamatorio multisistémico (SIM) debe ser considerado más allá de su baja prevalencia por su gravedad. ${ }^{1}$ El conocimiento del SIM basado en las características clínicas, los factores asociados a gravedad y las intervenciones terapéuticas realizadas, como se describe en el artículo, es de importancia vital para el reconocimiento precoz y el tratamiento inmediato. Es por ello por lo que la presentación y revisión bibliográfica que nos ofrecen los autores contribuye ampliamente a nuestra tarea asistencial. Un dato para considerar es que, si bien se trata de pacientes críticos, la tasa de mortalidad es baja, a la que también se hace referencia en el artículo.

La información abrumadora del COVID y del síndrome en cuestión (SIM) nos lleva a reflexionar sobre diferentes aspectos que, a nuestro entender, deben ser mencionados para evitar confusión. En este sentido, el primero para considerar es la relación entre la entidad (SIM) y las características clínicas que comparte con la enfermedad de Kawasaki (EK). ${ }^{2}$

Reportes europeos han mencionado un incremento de hasta 30 veces de la incidencia de EK en la misma época estacional en relación con los datos históricos (prepandemia). ${ }^{3}$ Estos pacientes con EK (que cumplen con los criterios diagnósticos) no presentaban diferencias con las características ampliamente conocidas de la EK. Sin embargo, existe un subgrupo de pacientes de mayor edad (> 5 años), manifestaciones más frecuentes gastrointestinales, neurológicas, miocarditis, falla hemodinámica y en cuanto al laboratorio (linfopenia, plaquetopenia, ferritina aumentada) que se han asociado a cuadros de mayor gravedad, considerados EK atípica. ${ }^{4,5}$
Aquellos pacientes con EK pertenecientes al subgrupo con las características descritas son más propensos a desarrollar síndrome shock Kawasaki (alrededor de un $10 \%$ desarrollarán SSK) o presentar un fenotipo similar al síndrome de shock tóxico. ${ }^{6}$ Estas dos últimas entidades mencionadas comparten características clínicas y bioquímicas con el SIM. Además, el $25 \%$ de los pacientes definidos como SIM pueden cumplir criterios diagnósticos de EK, aunque otras series demuestran hasta un $50 \%$ de pacientes con SIM que cumplen criterios de EK. Se podría hipotetizar que la raza, la etnia y la epigenética jugarían un rol en la relación virus-huésped que desarrollará un fenotipo u otro.

No se conoce la causa de estos cuadros graves, aunque se mencionan algunas hipótesis en la bibliografía en relación con la fisiopatología. La puesta en marcha del sistema inmune en presencia de un antígeno inicia un proceso de activación que comienza con la presentación antigénica por las células presentadoras de antígeno (CPA) a las células CD8+ y NK, lo que genera inflamación con liberación de citoquinas y quemoquinas, y activa el sistema inmune innato en primer lugar y luego el adaptativo. Pero, en diversas situaciones en las que podrían existir mecanismos virales de evasión de la respuesta inmunitaria, se origina una pérdida del equilibrio con tendencia a la desregulación inmune, lo que genera persistencia antigénica por falta de clearance viral, exageración de la respuesta inmune frente al estímulo y persistencia de células inflamatorias con la consiguiente liberación de citoquinas proinflamatorias, como factor de necrosis tumoral (tumor necrosis factor; $T N F$, por sus siglas en inglés) alfa, interferón gama, interleuquina 1 (IL-1), interleuquina 6 (IL-6) e interleuquina 18 (IL-18), entre otras.

La perpetuación de estas, se ve también acompañada de la acción de macrófagos activados que retroalimentan la respuesta bajo la generación de proteína inflamatoria de los macrófagos 1 beta (macrophage inflammatory protein-1; MIP-1 Beta, por sus siglas en inglés). ${ }^{7}$ Se produce, así, 
la llamada tormenta de citoquinas, considerada una reminiscencia de la linfohistiocitosis hemofagocítica hereditaria (HLH) y síndrome de activación macrófagica (SAM) u otros considerados bajo el paraguas de síndromes de tormentas de citoquinas, entre los que también se incluyen síndrome de distress respiratorio agudo, aquellos relacionados con malignidad, con infecciones, como virus de Epstein-Barr (Epstein-Barr virus; $E B V$, por sus siglas en inglés), influenza, y se incorpora actualmente COVID como un nuevo agente infeccioso capaz de gatillar la tormenta mencionada. ${ }^{8}$

Otro aspecto para tener presente es la relación temporal entre la infección y el desarrollo de SIM. Como describe el artículo de Taffarel y col., la evidencia de infección pasada con un intervalo de entre 2 y 6 semanas es el dato más encontrado en la bibliografía. La evidencia de infección presente o pasada (reactiva) sería otro aspecto para tener en cuenta en la toma de decisiones terapéuticas.

Hasta la fecha no hay datos relevantes sobre la predisposición de ciertas condiciones o patologías de base en pediatría, que permitan o faciliten el desarrollo de SIM. En cuanto a la criticidad, alrededor del $40 \%$ de los pacientes fueron admitidos en la Unidad de Cuidados Intensivos (UCI); el tiempo medio de estadía fue de 5 días; el $80 \%$ de los pacientes presentaban más de dos órganos de choque afectados, lo que hacía recordar el shock séptico y su manejo inicial a cargo de personal de salud especializado en el paciente crítico. ${ }^{6-9}$

La evidencia de tratamiento es de muy baja calidad limitada a reportes de casos, consensos o guías de expertos, lo cual no permite establecer opinión sobre la efectividad y la seguridad. De todas maneras, ante la falta de evidencia directa y estudios controlados, y teniendo en cuenta la similitud con enfermedades conocidas en las que ya se ha establecido la eficacia de ciertos tratamientos, se considera el uso de gammaglobulina endovenosa (GGEV), corticoides y agentes biológicos en el marco de decisiones compartidas por un equipo interdisciplinario de atención del paciente grave con SIM. ${ }^{10}$

El conocimiento y el reconocimiento de la entidad, como la conformación de equipos de atención para tomar decisiones y elaborar guías en nuestro medio o adaptar aquellas ya existentes, son los puntos claves del trabajo que permitirá que todo niño grave con diagnóstico de SIM reciba la mejor calidad de atención que, como pediatras, podemos brindarles.

Dra. María M. Katsicas, Reumatóloga pediatra, Servicio de Inmunología y Reumatología, Hospital de Pediatría "Prof. Dr. Juan P. Garrahan"

http:/ / dx.doi.org/10.5546/ aap.2021.4

Texto completo en inglés:

http:// dx.doi.org/10.5546/aap.2021.eng.4

Cómo citar: Katsicas MM. Tormenta de citoquinas y tormenta de información asociadas a COVID-19: consideraciones sobre el síndrome inflamatorio multisistémico en niños. Arch Argent Pediatr 2021;119(1): 4-5.

\section{REFERENCIAS}

1. Riphagen S, Gomez X, Gonzalez-Martinez C, Wilkinson $\mathrm{N}$, et al. Hyperinflammatory shock in children during COVID-19 pandemic. Lancet. 2020;395(10237):1607-8.

2. Toubiana J, Poirault C, Corsia A, Bajolle F, et al. Kawasakilike multisystem inflammatory syndrome in children during the covid-19 pandemic in Paris, France: prospective observational study. BMJ. 2020;369:m2094.

3. Verdoni L, Mazza A, Gervasoni A, Martelli L, et al. An outbreak of severe Kawasaki-like disease at the Italian epicentre of the SARS-CoV-2 epidemic: an observational cohort study. Lancet. 2020;395(10239):1771-8.

4. Pouletty M, Borocco C, Ouldali N, Caseris M, et al. Paediatricmultisystem inflammatory syndrome temporally associated withSARS-COV-2 mimicking Kawasaki disease (Kawa -COVID-19): a multicentre cohort. Ann Rheum Dis.2020;79(8):999-1006.

5. Whittaker E, Bamford A, Kenny J, Kaforou M, et al. Clinical Characteristics of 58 Children With a Pediatric Inflammatory Multisystem Syndrome Temporally Associated With SARS-CoV-2. JAMA. 2020;324(3):259-69.

6. Dallan C, Romano F, Siebert J, Politi S, et al. Septic shock presentation in adolescents with COVID-19. Lancet Child Adolesc health. 2020;4(7):e21-3.

7. Coperchini F, Chiovato L, Croce L, Magri F, et al. The cytokine storm in COVID-19: An overview of the involvement of the chemokine/chemokine-receptor system. Cytokine Growth Factor Rev. 2020;53:25-32.

8. Henderson L, Canna S, Schukert G, Volpi S, et al. On the alert for cytokine Storm: Immnopathology in COVID-19. Arthritis Rheumatol. 2020;72(7):1059-63.

9. Godfred-Cato S, Bryant B, Leung J, Matthew E, et al. COVID-19-Associated Multisystem Inflammatory Syndrome in Children — United States, March-July 2020. MMWR Morb Mortal Wkly Rep. 2020;69(32):1074-80.

10. American College of Rheumatology. Clinical Guidance for Pediatric Patients with Multisystem Inflammatory Syndrome in Children (MIS-C) Associated with SARSCoV-2 and Hyperinflammation in COVID-19. [Consulta: 14 de septiembre de 2020]. Disponible en: https:/ / www. rheumatology.org / Portals / 0 / Files / ACR-COVID-19Clinical-Guidance-Summary-MIS-C-Hyperinflammation. pdf. 